\title{
Liquid-Liquid Equilibrium of Aqueous Polymer Two-Phase Systems Using the Modified Wilson Equation
}

\author{
Pedro P. Madeira, ${ }^{\dagger, \ddagger}$ Xin Xu, ${ }^{\dagger}$ You-Ting Wu, José A. Teixeira, ${ }^{\ddagger}$ and \\ Eugénia A. Macedo ${ }^{\dagger, *}$
}

\begin{abstract}
Laboratory of Separation and Reaction Engineering, Departamento de Engenharia Química, Faculdade de Engenharia, Rua Dr. Roberto Frias, 4200-465, Porto, Portugal, and Centro de Engenharia Biológica, Universidade do Minho, 4710-057 Braga, Portugal
\end{abstract}

The modified Wilson equation, developed in a previous publication, for the excess Gibbs energy of aqueous polymer solutions, is extended to multicomponent mixtures. The model provides a flexible framework to correlate and predict phase equilibria of aqueous polymer two-phase systems. The results obtained with the model are in fair agreement with the experimental data.

\section{Introduction}

Beijerink $^{1}$ was the first to report on aqueous twophase systems (ATPS). It took around 50 years before these systems became popular, with the efforts of Albertsson, ${ }^{2}$ who applied them for separation processes. In recent years, the aqueous two-phase extraction has gained increased attention for purification and separation purposes particularly in the biotechnology field. ${ }^{3}$ A large number of publications have been reported concerning a wide range of applications of this method, including the separation of macromolecules, cell organelles, and viruses. ${ }^{3-5}$

The most widely studied systems are those composed of poly(ethylene glycol) (PEG) and dextran (Dex), or by PEG and potassium phosphate. ${ }^{6}$ Regardless of the potential, the ATPS application is limited by the high cost of the polymers commonly used in phase separation. The chemical costs can be as high as $74 \%$ of the total production price. ${ }^{7}$ There are in the literature several alternative systems in order to overcome this drawback. ${ }^{8-10}$ Among these, the use of thermoseparating polymers seems to be one of the most promising approaches. ${ }^{11-13}$

For the industrial success of a separation technique, it is advantageous to have mathematical tools accurately describing the thermodynamic properties of the process-associated systems. Their predictive ability is fundamental where experimental data is not available. There are in the literature principally two kinds of models describing ATPS: the osmotic virial-expansion models and those that lay on the lattice theory. ${ }^{14-18}$ Despite the relative practical success obtained with some of the foregoing models, they sometimes exhibit low accuracy in simultaneously predicting the phase diagrams of homologous ATPS (that differ only in the polymer molecular weight), and in some cases, they utilize different sets of model parameters to predict homologous ATPS. There is no such general solutions theory, and the development of new models may assist in understanding the experimental results observed in these complex systems. In a previous paper Xu et al. ${ }^{19}$ presented a new modified Wilson equation, to represent the vapor-liquid equilibrium (VLE) behavior of homologous aqueous polymer solutions, that incorporates

* Corresponding author. Tel: 351-22-5081653, Fax: 35122-5081674. E-mail address: eamacedo@fe.up.pt.

† Universidade do Porto.

† Universidade do Minho. some ideas from previous models, but in which the heat capacity is taken into account. Here we extended it to multicomponent systems to test its ability in correlating and predicting the LLE of polymer-polymer-water aqueous two-phase systems.

\section{Model Development}

As usual the model development starts with the excess Gibbs energy $G^{E}$ (eq 1 ). In this new modified Wilson equation the excess heat capacity, $c_{P}^{E}$, that provides an important link between the excess enthalpy and the excess entropy (eqs 2 and 3 ) is used:

$$
\begin{aligned}
G^{E} & =H^{E}-T S^{E} \\
c_{P}^{E} & =\left(\frac{\partial H^{E}}{\partial T}\right)_{P, x} \\
c_{P}^{E} & =T\left(\frac{\partial S^{E}}{\partial T}\right)_{P, x}
\end{aligned}
$$

The expression for the excess Gibbs energy can be easily obtained (Xu et al.): ${ }^{19}$

$$
\frac{G^{E}}{R T}=-\frac{S_{C}^{E}}{R}+\frac{H^{E}}{R T}-\frac{1}{R} \int_{\infty}^{T} \frac{1}{T}\left(\frac{\partial H^{E}}{\partial T}\right)_{P, x} d T
$$

The first term is called the combinatorial contribution: it is independent of temperature and accounts for the size/shape of the molecules; the last terms both depend on temperature and are called the residual contribution and reflect the interactions between segments of molecules. Therefore, the model consists of a combinatorial term $G_{c}^{E}$ and a residual term $G_{R}^{E}$ :

$$
\begin{gathered}
\frac{G_{R}^{E}}{R T}=\frac{H^{E}}{R T}-\frac{1}{R} \int_{\infty}^{T} \frac{1}{T}\left(\frac{\partial H^{E}}{\partial T}\right)_{P, x} d T \\
\frac{G_{C}^{E}}{R T}=-\frac{S_{C}^{E}}{R}
\end{gathered}
$$

The excess is defined on an asymmetric normalization: for water the reference state is the pure liquid and for the solutes it is a hypothetical liquid (one mole of solution in pure water, with interactions such as in an infinite diluted aqueous solution). The principles and methodologies adopted for the model development are 
given in the previous paper for binary mixtures $(\mathrm{Xu}$ et al. ${ }^{19}$ The extension to multicomponent mixtures is straightforward, the expressions for the combinatorial and residual contributions for the excess Gibbs energy being as follows:

$$
\begin{gathered}
\frac{G_{C}^{E}}{R T}=\sum_{i=1}^{m} n_{i} \ln \frac{X_{i}}{x_{i}}+\frac{1}{\alpha} \sum_{i=1}^{m} q_{i} n_{i} \ln \frac{X_{i}}{\Phi_{i}} \\
\frac{G_{R}^{E}}{R T}=-\frac{n_{q}}{\alpha}\left[\sum_{i=1}^{m} X_{i} \ln \left(\sum_{j=1}^{m} X_{j} G_{j i}\right)\right]
\end{gathered}
$$

where $X_{i}=\theta_{i}$, i.e., the effective mole fraction of a segment is equal to the surface/area fraction:

$$
\begin{array}{ll}
N_{r}=\sum_{i=1}^{m} N_{i} r_{i}, & \Phi_{i}=N_{i} r_{i} / N_{r} \\
N_{q}=\sum_{i=1}^{m} N_{i} q_{i}, & X_{i}=N_{i} q_{i} / N_{q}
\end{array}
$$

$n_{i}$ and $N_{i}$, are the mole numbers and molecule numbers of species $i$, respectively; $r_{i}$ are the numbers of segments per molecule. The $\Phi_{i}$ are volume fractions; $q_{i}$ means effective segment number of species $i$ and is correlated in the usual way,

$$
q_{i}=\left[r_{i}(z-2)+2\right] / z=r_{i}\left[1-2\left(1-1 / r_{i}\right) / z\right]
$$

The structural factor of the solution $2 / z$ is defined as the nonrandom factor $\alpha$. The typical value is $0.3 .^{20}$ Thus,

$$
G_{i j}=\exp \left(-\alpha \tau_{i j}\right)
$$

where $\tau_{i j}$ are the interaction parameters:

$$
\tau_{i j}=\frac{1}{\alpha} \frac{\epsilon_{i j}-\epsilon_{j j}}{R T}
$$

and $q_{i}$ is rewritten as

$$
q_{i}=r_{i}\left[1-\alpha\left(1-1 / r_{i}\right)\right]
$$

Using standard thermodynamics, the activity coefficients can be easily obtained:

$$
\begin{gathered}
\ln \gamma_{i}=\ln \gamma_{i}^{C}+\ln \gamma_{i}^{R} \\
\ln \gamma_{i}^{C}=\ln \frac{X_{i}}{x_{i}}+\sum_{j=1}^{m} X_{j}\left(1-\frac{q_{i}}{q_{j}}\right)+\frac{q_{i}[}{\alpha} \ln \frac{X_{i}}{\Phi_{i}}+ \\
\left.\sum_{j=i}^{m} \Phi_{j}\left(\frac{q_{j} r_{i}}{q_{i} r_{j}}-1\right)\right] \\
\ln \gamma_{i}^{R}=-\frac{q_{i}}{\alpha}\left[\ln \left(\sum_{j=1}^{m} X_{j} G_{j i}\right)+\sum_{k=1}^{m} \frac{X_{k} G_{i k}}{\sum_{j=1}^{m} X_{j} G_{j k}}-1\right]
\end{gathered}
$$

The activity coefficients are calculated, according to eqs $13 \mathrm{a}-\mathrm{c}$, in the mole fraction scale, and the variables have the meanings already presented. The activity coefficients of the polymers were normalized to the reference state in ATPS. Thus, according to the nonsymmetric convention,

$$
\ln \gamma_{i}^{*}=\ln \gamma_{i}-\ln \gamma_{i}^{r e f}
$$

where $\ln \gamma_{i}^{r e f}$ is the activity coefficient in the reference state and is calculated from eq 13 , in which $x_{1}=1$ and $x_{i}=0(i \neq 1)$, ( 1 stands for solvent and $i \neq 1$ for solutes $)$.

The dependence of the interaction parameters, between water and segment of polymer and between the segments of polymers with the temperature, are, according to the suggestion of $\mathrm{Wu},{ }^{17}$ as follows:

$$
\begin{gathered}
\tau_{j i}=a_{j i}^{(1)}\left(T_{0} / T\right)+a_{j i}^{(2)}\left(T_{0} / T\right)^{2} \\
\tau_{i j}=a_{i j}^{(1)}\left(T_{0} / T\right)+a_{i j}^{(2)}\left(T_{0} / T\right)^{2} \\
a_{j i}^{(1)}=\frac{z}{2}\left[\frac{\epsilon_{j i}-\epsilon_{i i}}{k T_{0}}+\left(\frac{\delta_{j i}}{1+q_{j i}}-\frac{\delta_{i i}}{1+q_{i i}}\right) / T_{0}\right] \\
a_{j i}^{(2)}=-\frac{z}{2}\left[\frac{q_{j i}}{2\left(1+q_{j i}\right)^{2}}\left(\frac{\delta_{j i}}{k T_{0}}\right)^{2}-\frac{q_{i i}}{2\left(1+q_{i i}\right)^{2}}\left(\frac{\delta_{i i}}{k T_{0}}\right)^{2}\right]
\end{gathered}
$$

where $\epsilon$ is the interaction energy between segmentsegment pairs; $\delta$ is the oriented interaction parameter between segment-segment pairs and $q$ is the ratio of statistical degeneracy of two states. $a_{i j}^{(1)}$ and $a_{i j}^{(2)}$ can be expressed in a similar way to $a_{j i}^{(1)}$ and $a_{j i}^{(2)}$ replacing the underscripts $i$ by $j$, and $j$ by $i$. $T_{0}$ is the reference temperature, $T_{0}=298.15 \mathrm{~K}$. According to Xu et al. ${ }^{19} \mathrm{it}$ is assumed that $a^{(1)}$ and $a^{(2)}$ are temperature and composition independent, and if the range of temperature is not too wide, the oriented interactions between the $i-i$ and $j-j$ pairs can be neglected $\left(\delta_{i i}=0\right)$. Thus, $a_{j i}^{(2)}=a_{i j}^{(2)}$.

\section{Results and Discussion}

To calculate the polymer-polymer interaction parameters, the component isoactivity criterion between the two-liquid phases is used:

$$
\left(x_{i} \gamma_{i}\right)^{I}=\left(x_{i} \gamma_{i}\right)^{I I}
$$

where $I$ and $I I$ represent both liquid phases. Therefore, the liquid-liquid data from the ternary systems water/ polymer/polymer is needed to estimate the parameters. For this purpose, LLE data measured in our laboratory ${ }^{9}$ as well as data collected from literature were used to test the new model. The phase forming polymers studied in this work can be divided in two categories: the classical polymers, like poly(ethylene glycol) (PEG) and dextran (Dex) and the polymers that have been recently used in ATPS and which exhibit thermoseparating properties, like PES (a water soluble starch polymer) and random copolymers of ethylene oxide (EO) and propylene oxide (PO) (symbolized as EOPO and Ucon). The working equations are eqs $13 a-c$. For the calculations, pure substance properties are necessary, such as the molecular weight $\left(M_{j}\right)$, the segment number $\left(r_{j}\right)$ and the molar volume $\left(V_{j}\right)$. The $M_{j}$ was set equal to the polymer number average molecular weight $\left(M_{n}\right)$. Since the van der Waals volume of a repeated unit in PEG molecule is very close to the double of a water molecule, $V_{\text {peg }}$ is approximated as $\left(2 n_{\text {peg }}+1\right) V_{w}$, where $n_{\text {peg }}$ is the polymerization degree of a PEG molecule and can be 
Table 1. Modified Wilson Parameters for PEG-Dex and EOPO-PES ATPS

\begin{tabular}{cccccr}
\hline \multicolumn{1}{c}{ system } & $T(\mathrm{~K})$ & $\alpha_{23}{ }^{a}$ & $\tau_{32}$ & $\tau_{23}$ & ref \\
\hline PEG6000-Dex40 & 298.15 & 0.3 & -1.104 & 1.557 & 5 \\
PES100-EOPO(1:1) & 298.15 & 0.3 & -3.282 & 46.42 & 16
\end{tabular}

${ }^{a}$ Fixed at 0.30 .

calculated from $M_{\text {peg. }}{ }^{18}$ Similarly, for EOPO, $V_{\text {eopo }}$ is approximated as $\left(5 n_{\text {eopo }}+1\right) V_{w}$. For Dex, $V_{\text {dex }}$ is calculated from its specific volume $\left(v_{d e x}\right), v_{d e x}=5.96 \times$ $10^{-4} \mathrm{~m}^{3} \mathrm{~kg}^{-1}$ at $293.15 \mathrm{~K} .{ }^{18}$ For water, $r_{w}=1, V_{w}=18.05$ $\times 10^{-6} \mathrm{~m}^{3} \mathrm{~mol}^{-1}$ at $298.15 \mathrm{~K}^{18}$ The same value is used at other temperatures. Thus, $r_{2}=V_{2} / V_{1}$. For PES $r_{\text {pes }}$ was set equal to the polymerization degree of a PES molecule, $n_{\text {pes }}$.

Since the model describes satisfactorily the VLE using only two parameters, i.e., $a_{j i}^{(2)}=a_{i j}^{(2)}=0,{ }^{19}$ in the LLE data treatment the oriented interactions were not accounted. Thus, it is only necessary to estimate the parameters $\tau_{i j}$ and $\tau_{j i}$, which reflect the interactions between the polymers segments. For its calculation, the LLE data were adjusted using the following objective function, F, based on the isoactivity criteria, and used by other authors for the treatment of this kind of systems: ${ }^{21-23}$

$$
\begin{aligned}
F=\sum_{j}\left[\frac{\left(x_{1} \gamma_{1}^{*}\right)_{j}^{I}-\left(x_{1} \gamma_{1}^{*}\right)_{j}^{I I}}{\left(x_{1} \gamma_{1}^{*}\right)_{j}^{I I}}\right]+ & \sum_{j}\left[\frac{\left(x_{2} \gamma_{2}^{*}\right)_{j}^{I}-\left(x_{2} \gamma_{2}^{*}\right)_{j}^{I I}}{\left(x_{2} \gamma_{2}^{*}\right)_{j}^{I I}}\right]+ \\
& \sum_{j}\left[\frac{\left(x_{3} \gamma_{3}^{*}\right)_{j}^{I}-\left(x_{3} \gamma_{3}^{*}\right)_{j}^{I I}}{\left(x_{3} \gamma_{3}^{*}\right)_{j}^{I I}}\right]
\end{aligned}
$$

where I and II refer to both phases, and $\mathrm{j}$ is the number of tie-lines.

The modified Wilson model parameters, that reflect the interactions between the polymer-polymer segments, were obtained using the simplex method of Nelder and Mead. ${ }^{24}$ Only two tie-lines were used to estimate the parameters. The other tie-lines for the same system, as well for other systems with different polymer molecular weight, were predicted using the same parameters, according to the equality of the activities of the components in both phases. This methodology has already been successfully used in our group to correlate and predict the LLE of polymer-salt/ water aqueous two-phase systems. ${ }^{25}$

Table 1 presents the polymer-polymer interaction parameters for the PEG-Dex and EOPO-PES ATPS. Table 2 summarizes the absolute deviations for the

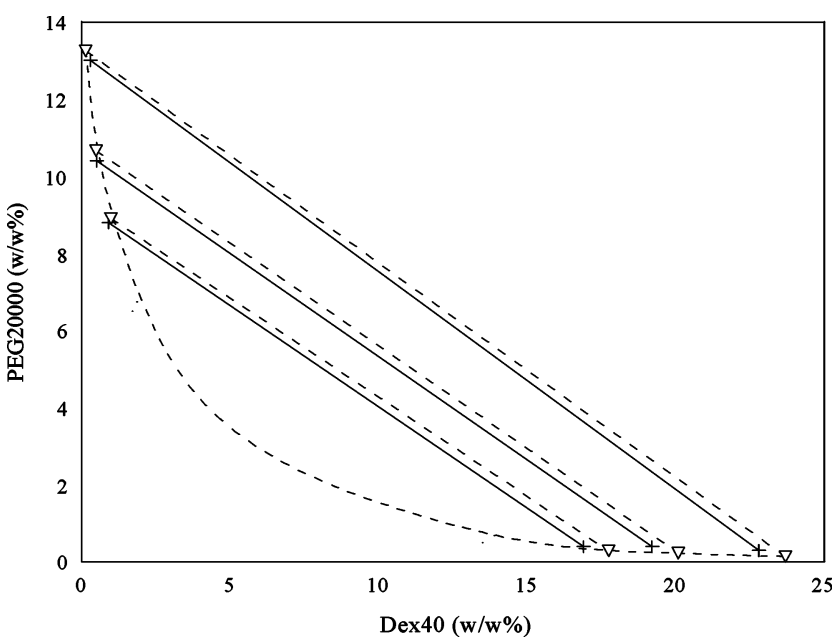

Figure 1. Phase diagram of PEG20000-Dex40 ATPS at 298.15 K. $(+-+)$ experimental data and tie-lines; $(\nabla--\nabla)$ predicted tielines; (- -) predicted binodal. (Predictions with the PEG-Dex interaction parameters presented in Table 1).

ATPS studied, as well as the source of the experimental data used. Figures 1 to 5 show some comparisons between experimental and predicted phase diagrams.

The expressions for the calculation of $\delta W_{i}^{\alpha}, \delta W$ and $\delta W_{\max }$ are as follows:

$$
\begin{gathered}
\delta W_{i}^{\alpha}=\frac{\sum_{j=1}^{N} \mid W_{j, i}^{\alpha}(\text { calc. })-W_{j, i}^{\alpha}(\exp .) \mid}{N} \\
\delta W=\frac{\sum_{\alpha} \sum_{i} \delta W_{i}^{\alpha}}{2 N_{1}} \\
\delta W_{\max }=\max \left\{\mid W_{j, i}^{\alpha}(\text { calc. })-W_{j, i}^{\alpha}(\exp .) \mid\right\}
\end{gathered}
$$

where $\delta W_{i}^{\alpha}, \delta W$ and $\delta W_{\max }$ mean the average absolute deviation in weight percentage of component $i$ in phase $\alpha$, the overall average absolute deviation and the maximum absolute deviation, respectively. $N$ is the number of experimental data points and $N_{1}$ the number of components in the system.

\begin{tabular}{|c|c|c|c|c|c|c|c|c|c|}
\hline \multirow[b]{2}{*}{ system } & \multirow[b]{2}{*}{$T(\mathrm{~K})$} & \multirow[b]{2}{*}{$\delta W_{2}^{T(e)}$} & \multirow[b]{2}{*}{$\delta W_{3}^{T(e)}$} & \multirow[b]{2}{*}{$\delta W_{2}^{B(e)}$} & \multirow[b]{2}{*}{$\delta W_{3}^{B(e)}$} & \multirow[b]{2}{*}{$\delta W$} & \multirow[b]{2}{*}{$\delta W_{\max }$} & \multicolumn{2}{|c|}{$\mathrm{STL}^{(f)}$} \\
\hline & & & & & & & & exp. & pred. \\
\hline PEG6000-Dex40(a) & 298.15 & 0.58 & 0.42 & 0.24 & 1.81 & 0.97 & 2.28 & -0.50 & -0.50 \\
\hline PEG8000-Dex40 & 295.15 & 0.18 & 0.13 & 0.13 & 0.34 & 0.22 & 0.66 & -0.53 & -0.49 \\
\hline PEG10000-Dex40 ${ }^{(a)}$ & 298.15 & 0.09 & 0.18 & 0.29 & 1.50 & 0.58 & 1.74 & -0.51 & -0.50 \\
\hline PEG20000-Dex40 ${ }^{(a)}$ & 298.15 & 0.07 & 0.06 & 0.35 & 1.89 & 0.67 & 2.05 & -0.53 & -0.50 \\
\hline PEG10000-Dex110 $(a)$ & 313.15 & 0.51 & 0.03 & 0.16 & 0.92 & 0.53 & 1.19 & -0.55 & -0.49 \\
\hline PEG6000-Dex70 ${ }^{(a)}$ & 296.15 & 0.45 & 0.06 & 0.22 & 0.64 & 0.46 & 0.95 & -0.55 & -0.50 \\
\hline PEG6000-Dex110 ${ }^{(a)}$ & 298.15 & 0.04 & 0.12 & 0.26 & 0.37 & 0.23 & 1.24 & -0.48 & -0.47 \\
\hline PEG6000-Dex500 ${ }^{(a)}$ & 298.15 & 0.08 & 0.20 & 0.28 & 1.47 & 0.38 & 2.18 & -0.46 & -0.44 \\
\hline $\operatorname{EOPO}(1: 1)-P E S 100(b)$ & 298.15 & 0.61 & 0.47 & 0.63 & 0.62 & 0.61 & 1.94 & -0.54 & -0.60 \\
\hline $\operatorname{EOPO}(1: 1)-P E S 200^{(b)}$ & 298.15 & 0.14 & 0.19 & 0.45 & 0.60 & 0.29 & 2.13 & -0.48 & -0.48 \\
\hline Ucon-PES100 ${ }^{(c)}$ & 295.15 & 1.57 & 0.98 & 0.71 & 0.66 & 0.93 & 2.08 & -0.46 & -0.57 \\
\hline
\end{tabular}

From Table 2 it is possible to conclude that the predicted results are in fair agreement with the experimental data. In fact, all $\delta W$ and almost all $\delta W_{i}^{\alpha}$ values

Table 2. Comparison of Predicted Results and Experimental Data for PEG-Dex and EOPO-PES ATPS

Data from: ${ }^{(a)}(5) ;{ }^{(b)}(16) ;{ }^{(c)}(9) ;{ }^{(d)}(4) .{ }^{(e)}$ The 2 component is predominant in the top phase, i.e., PEG and EOPO, and the 3 predominates in the bottom phase, i.e., Dex and PES. T and B mean top and bottom phase, respectively. (f) Slope of Tie Line. 


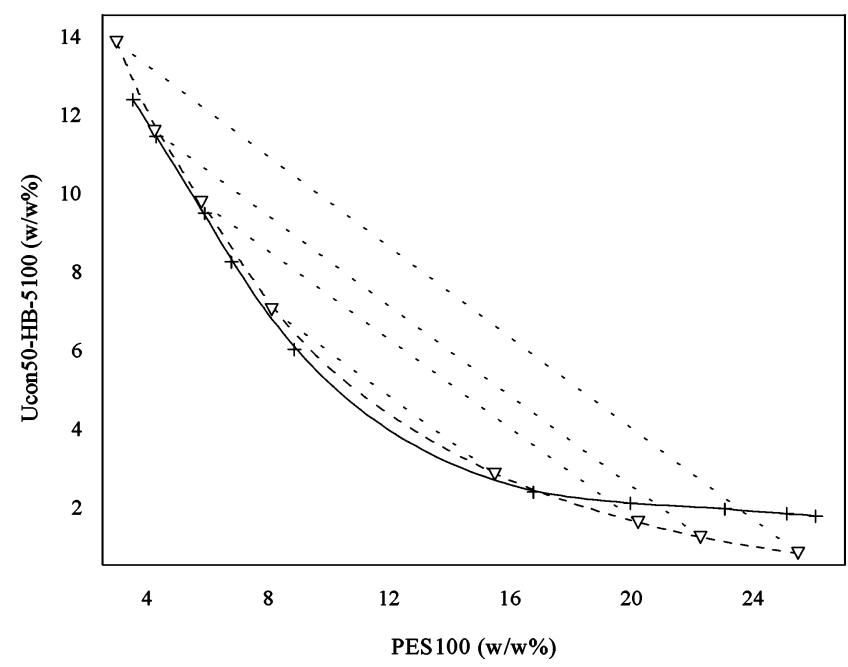

Figure 2. Phase diagram of Ucon-PES100 ATPS at $295.15 \mathrm{~K}$. $(+-+)$ experimental data and binodal; $(\nabla---\nabla)$ predicted tie-lines; (- - -) predicted binodal.

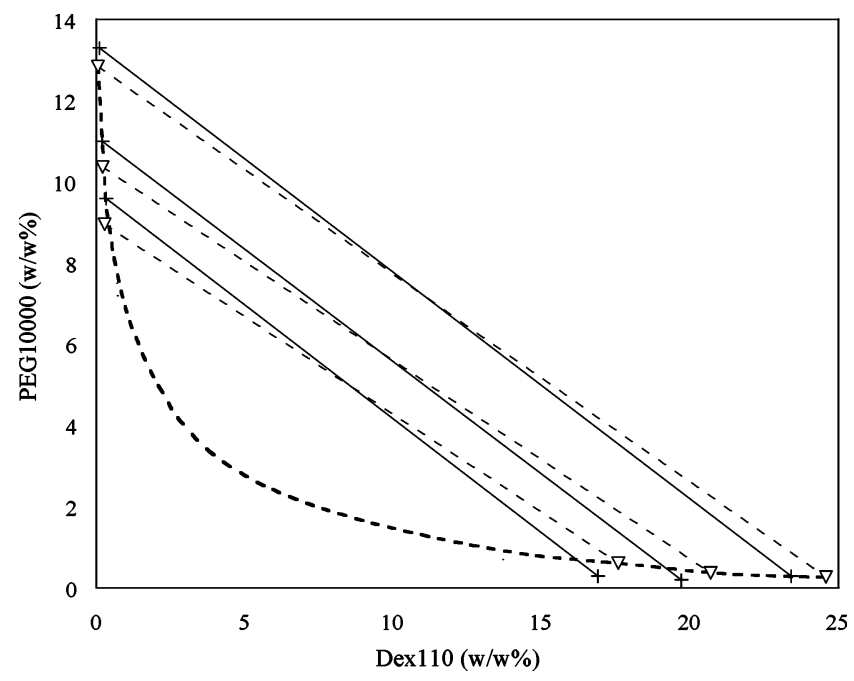

Figure 3. Phase diagram of PEG10000-Dex110 ATPS at 313.15 K. $(+-+)$ experimental data and tie-lines; $(\nabla--\nabla)$ predicted tielines; (- -) predicted binodal. (Predictions with the PEG-Dex interaction parameters presented in Table 1).

are smaller, in weight percentage, than 1.0 and $\delta W_{\max }$ assume values lower than 2.3. Thus, the model can predict accurately several phase diagrams in which the polymers differ only in the molecular weight, as can also be observed from Figure 1.

Although the Ucon and EOPO (1:1) polymers are slightly different, (the molar ratio in EO:PO is 1:1 in the polymer used in the correlation of the parameters, and approximately $1.3: 1$ in the Ucon), the prediction results are very satisfactory. Such fact explains the difference between the slope of the tie line (STL) and between the polymer concentrations experimentally obtained and calculated with the model (Figure 2 and Table 2).

The modified Wilson equation proves to be a powerful tool to correlate and predict homologous ATPS, even for systems at relatively high temperatures (Figure 3 ).

Regarding Figures 4 and 5, it is possible to see that the model also predicts accurately the influence of the molecular weight of the polymers on the phase diagram: increasing the polymer molecular weight the binodal moves towards the lower concentrations.

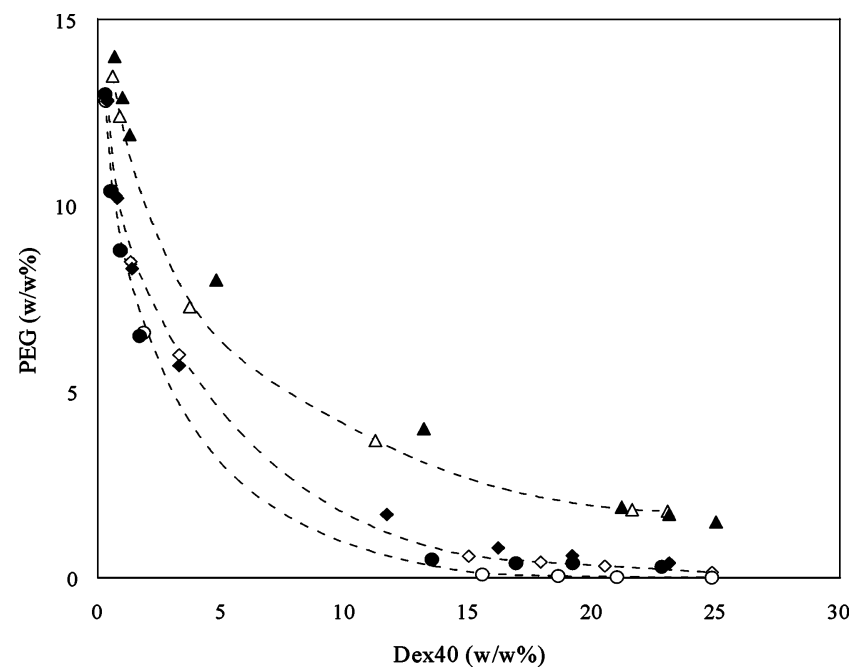

Figure 4. Influence of PEG's molecular weight in the PEG-Dex40 ATPS diagram at 298.15 K. Experimental data for (ム) PEG6000, $(\bullet)$ PEG10000, and (๑) PEG20000. Predicted binodals for $(\triangle---\triangle)$ PEG6000, $(\diamond---\diamond)$ PEG10000, and (O- - -O) PEG20000.

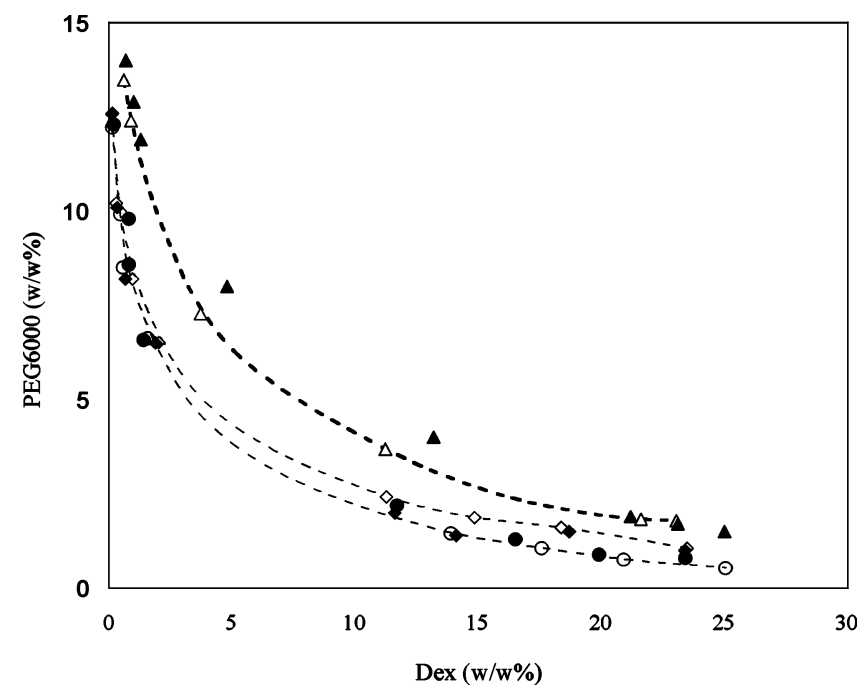

Figure 5. Influence of Dex's molecular weight in the PEG6000Dex ATPS diagram at 298.15 K. Experimental data for (ム) Dex40, $(\checkmark)$ Dex110, and $(\bullet)$ Dex500. Predicted binodals for $(\triangle--\triangle)$ Dex40, $(\diamond-$ - $\diamond$ Dex110, and (○- - - ) Dex500.

\section{Conclusions}

The modified Wilson equation was extended to describe the LLE in polymer/polymer/water ATPS in multicomponent systems. The results obtained show that the model is a powerful framework, both for correlation and for prediction of phase diagrams of homologous ATPS, even at relatively high temperatures. Moreover, the data indicate that only one set of parameters is enough to predict homologous ATPS.

\section{Acknowledgment}

Xin $\mathrm{Xu}$ acknowledges the postdoctoral fellowship provided by FCT (Fundação para a Ciência e a Tecnologia), Ministério da Ciência e Tecnologia, Portugal, and Pedro P. Madeira acknowledges the financial support from FCT (Project POCTI/2000/EQU/33185).

\section{List of Symbols}

$a=$ interaction parameter defined in eq 15 , or activity cal. $=$ calculated 
$c_{P}^{E}=$ excess heat capacity/heat capacity change of mixing

exp. $=$ experimental

$F=$ objective function

$G, G^{E}=$ binary parameter, excess Gibbs energy

$H^{E}=$ excess enthalpy

$K=$ Boltzmann constant

$M, M_{n}=$ molecular weight, number-average molecular weight

$n=$ mole number of segment-segment pairs, or polymerization degree

$N=$ number of molecules (segment-segment pairs), or experimental data points

$N_{1}=$ number of experimental data points

$P=$ pressure

Pred. $=$ prediction

$q=$ effective segment number of polymer or ratio of statistical degeneracy of two states

$r=$ number of segments per molecule

$R=$ gas constant

$S^{E}=$ excess entropy

$S S Q=$ sum of squares

$S T L=$ slope of tie line

$T=$ absolute temperature

$T_{0}=$ reference temperature, $298.15 \mathrm{~K}$

$V=$ molar volume

$W=$ weight fraction

$x=$ mole fraction of polymer solutions

$X=$ effective mole fraction of segments

$z=$ coordination number in the lattice theory

\section{Greek Letters}

$\alpha=$ nonrandom factor in the Wilson model

$\delta=$ deviation or oriented interaction parameter between segment-segment pairs

$\epsilon=$ interaction energy between segment-segment pairs

$\Phi=$ volume fraction

$\gamma=$ activity coefficient

$\theta=$ surface/area fraction

$\tau=$ binary interaction parameter

$v=$ specific volume

$\partial=$ partial derivative

$\infty=$ infinity

\section{Subscripts}

$C=$ combinatorial factor

$i, j=$ any species or segments

$i i, i j, j j=$ segment - segment pairs

$R=$ residual contribution

1, 2 = solvent and polymer, respectively

\section{Superscripts}

$C=$ combinatorial factor

$E=$ notation of excess quality

$R=$ residual factor

$r e f=$ reference state

(1), (2) = notation for distinction

$I, I I=$ any aqueous phase

\section{Literature Cited}

(1) Beijerinck, M. W. Über eine Eigentümlichkeit der löslichen Stärke. Centralbl Bakt. 1896, 2, 679-699.

(2) Albertsson, P.-Å. Chromatography and partition of cells and cell fragments. Nature 1956, 177, 771.

(3) Hustedt, H.; Kroner, K. H.; Kula, M. R. Applications of Phase Partitioning in Biotechnology. In Partitioning in aqueous two-phase systems. Theory, methods, uses, and applications to biotechnology; Walter, H., Brooks, D. E., Fisher, D., Eds.; Academic Press: London, 1985.

(4) Albertsson, P.-Å Partition of cell particles and macromolecules, 3rd ed.; New York: John Wiley \& Sons, 1986.
(5) Zaslavsky, B. Y. Aqueous two-phase partitioning; Marcel Dekker: New York, 1995.

(6) Huddleston, J.; Veide, A.; Köhler, K.; Flanagan, J.; Enfors, S. O.; Lyddiatt, A. The molecular basis of partitioning in aqueous two-phase systems. Trends Biotechnol. 1991, 9, 381.

(7) Datar, R. Economics of primary separation steps in relation to fermentation and genetic engineering. Process Biochem. 1986, $21,19$.

(8) Kroner, K. H.; Hustedt, H.; Kula, M. R. Evaluation of crude dextran as a phase forming polymer for the extraction of enzymes in aqueous two-phase systems in large scale. Biotechnol Bioeng. 1982, 24, 1015.

(9) Pereira, M.; Wu, Y.-T.; Madeira, P. P.; Venâncio, A.; Macedo, E. A.; Teixeira, J. Liquid-Liquid Equilibrium Phase Diagrams of New Aqueous Two-Phase Systems: Ucon 50-HB5100 - Ammonium Sulfate, Ucon 50-HB5100 - Poly(vinyl alcohol), Ucon 50HB5100 - Hydroxypropyl Starch and Polyethylene Glycol 8000 - Polyvinyl Alcohol. J Chem. Eng. Data 2004, 49, 43.

(10) Venâncio, A.; Teixeira, J. A.; Mota, M. Evaluation of crude hydroxypropyl starch as a bioseparation aqueous phase forming polymer. Biotechnol Progr. 1993, 9, 635.

(11) Johansson, H. O.; Lundh, G.; Karlström, G.; Tjerneld, F. Effects of ions on partitioning of serum albumin and lysozyme in aqueous two-phase systems containing ethylene oxide/propylene oxide co-polymers. Biochim. Biophys. Acta 1996, 1290, 289.

(12) Pereira, M.; Wu, Y.-T.; Venâncio, A.; Teixeira, J. Aqueous Two-Phase Extraction Using Thermoseparating Polymer: A New System for the Separation of Endo-polygalacturonase. Biochem Eng J. 2002, 3691, 1.

(13) Persson, J.; Johansson, H. O.; Tjerneld, F. Purification of protein and recycling of polymers in a new aqueous two-phase system using two thermoseparating polymers. J. Chromatogr. A 1999, 864, 31 .

(14) Grossmann, C.; Zhu, J.; Maurer, G. Phase equilibrium studies on two-phase systems containing amino acids and peptides. Fluid Phase Equilib. 1993, 82, 275.

(15) Kang, C. H.; Sandler, S. I. Phase behavior of aqueous twopolymer systems. Fluid Phase Equilibr. 1987, 38, 245.

(16) Li, M.; Zhu, Z.-Q.; Wu, Y.-T.; Lin, D.-Q. Measurement of phase diagrams for new aqueous two-phase systems and prediction by a generalized multicomponent osmotic virial equation. Chem. Eng. Sci. 1998, 53, 2755.

(17) Wu, Y.-T.; Zhu, Z.-Q.; Lin, D.-Q.; Mei, L. H. A modified NRTL equation for the calculation of phase equilibrium of polymer solutions. Fluid Phase Equilibr. 1996, 121, 125.

(18) Wu, Y.-T.; Lin, D.-Q.; Zhu, Z.-Q. Thermodynamics of aqueous two-phase systems-the effect of polymer molecular weight on liquid-liquid equilibrium phase diagrams by the modified NRTL model. Fluid Phase Equilibr. 1998, 147, 25.

(19) Xu, X.; Madeira, P. P.; Teixeira, J. A.; Macedo, E. A. A New Modified Wilson Equation for the Calculation of Vapor-Liquid Equilibrium of Aqueous Polymer Solutions. Fluid Phase Equilibr. 2003, 213,53 .

(20) Prausnitz, J.; Lichtenthaler, R. N.; Azevedo, E. G. Molecular thermodynamics of fluid-phase equilibria, 3rd ed.; Prentice Hall PTR: Upper Saddle river, NJ, 1999.

(21) Gao, Y. L.; Peng, Q. H.; Li, Z. C.; Li, Y. G. Thermodynamics of ammonium sulfate-poly(ethylene glycol) aqueous two-phase systems. Part 1. Experiment and correlation using extended UNIQUAC equation. Fluid Phase Equilibr. 1991a, 63, 157.

(22) Gao, Y. L.; Peng, Q. H.; Li, Z. C.; Li, Y. G. Thermodynamics of ammonium sulfate-poly(ethylene glycol) aqueous two-phase systems. Part 2. Correlation and prediction using extended UNIFAC equation. Fluid Phase Equilibr. 1991b, 63, 173.

(23) Haghtalab, A.; Mokhtarani, B. On extension of UNIQUAC-NRF model to study the phase behavior of aqueous twophase polymer-salt systems. Fluid Phase Equilib. 2001, 180, 139.

(24) Nelder, J. A.; Mead, R. A. A simplex method for function minimization. Comput J. 1965, 7, 308.

(25) Xu, X.; Madeira, P. P.; Macedo, E. A. Representation of liquid-liquid equilibria for polymer-salt aqueous two-phase systems. Chem. Eng. Sci. 2004, 59, 1153.

Received for review September 6, 2004 Revised manuscript received December 15, 2004 Accepted January 18, 2005 